Acta Crystallographica Section D

\section{Biological Crystallography}

ISSN 1399-0047

\section{Emily Golden, Amir Karton and Alice Vrielink*}

School of Chemistry and Biochemistry, University of Western Australia, Crawley, Western Australia 6009, Australia

Correspondence e-mail:

alice.vrielink@uwa.edu.au

\title{
High-resolution structures of cholesterol oxidase in the reduced state provide insights into redox stabilization
}

Cholesterol oxidase ( $\mathrm{CO})$ is a flavoenzyme that catalyzes the oxidation and isomerization of cholesterol to cholest-4-en-3one. The reductive half reaction occurs via a hydride transfer from the substrate to the FAD cofactor. The structures of $\mathrm{CO}$ reduced with dithionite under aerobic conditions and in the presence of the substrate 2-propanol under both aerobic and anaerobic conditions are presented. The $1.32 \AA$ resolution structure of the dithionite-reduced enzyme reveals a sulfite molecule covalently bound to the FAD cofactor. The isoalloxazine ring system displays a bent structure relative to that of the oxidized enzyme, and alternate conformations of a triad of aromatic residues near to the cofactor are evident. A $1.12 \AA$ resolution anaerobically trapped reduced enzyme structure in the presence of 2-propanol does not show a similar bending of the flavin ring system, but does show alternate conformations of the aromatic triad. Additionally, a significant difference electron-density peak is observed within a covalent-bond distance of N5 of the flavin moiety, suggesting that a hydride-transfer event has occurred as a result of substrate oxidation trapping the flavin in the electron-rich reduced state. The hydride transfer generates a tetrahedral geometry about the flavin N5 atom. High-level densityfunctional theory calculations were performed to correlate the crystallographic findings with the energetics of this unusual arrangement of the flavin moiety. These calculations suggest that strong hydrogen-bond interactions between Gly120 and the flavin N5 centre may play an important role in these structural features.

\section{Introduction}

Cholesterol oxidase ( $\mathrm{CO})$ is a flavoenzyme that catalyzes the oxidation and isomerization of cholesterol to cholest-4-en-3one (Fig. 1). The type I CO from Streptomyces SA-COO (EC 1.1.3.6) is a member of the glucose-methanol-choline (GMC) oxidoreductase family and contains a single molecule of flavin adenine dinucleotide (FAD) noncovalently but tightly bound to the protein. The enzyme catalyzes the oxidation and isomerization of steroids containing a $3 \beta$-hydroxyl group, with the cholestane group conferring binding specificity in the active site (Pollegioni et al., 1999). CO is also able to oxidize small-molecule alcohols such as 2-propanol and methanol, with a preference for those containing aromatic rings; however, these molecules display a lack of specificity, resulting in a much slower rate of catalysis (Pollegioni et al., 1999).
Received 25 September 2014 Accepted 17 October 2014

PDB references: cholesterol oxidase, complex with 2-propanol, reduced state, $4 \mathrm{u} 2 \mathrm{~s}$; complex with 2-propanol, oxidized state, $4 \mathrm{u} 2 \mathrm{t}$; containing an FADsulfite adduct, $4 \mathrm{u} 2 \mathrm{l}$ 
Substrate oxidation results in reduction of the cofactor during the reductive half reaction. An oxidative half reaction occurs with re-oxidation of the flavin cofactor by molecular oxygen to form hydrogen peroxide (Fig. 1).

High-resolution X-ray structures (Chen et al., 2008; Lario et al., 2003; Lyubimov et al., 2006, 2007, 2009) along with mutagenesis studies (Kass \& Sampson, 1998; Sampson \& Kass, 1997; Yin et al., 2001, 2002; Yue et al., 1999) have revealed the key active-site residues that are responsible for performing the catalytic function. His447 is important for the oxidation reaction (Kass \& Sampson, 1998; Yamashita et al., 1998), with mutations of this residue significantly decreasing the rate of oxidation of cholesterol (more than 100000 times lower on mutation to lysine, glutamate, aspartate and alanine; Kass \& Sampson, 1998). Atomic resolution studies of the enzyme revealed that the NE2 atom of His447 was protonated and this led to the suggestion that His 447 functions to position the substrate with respect to the flavin and an active-site gluta-

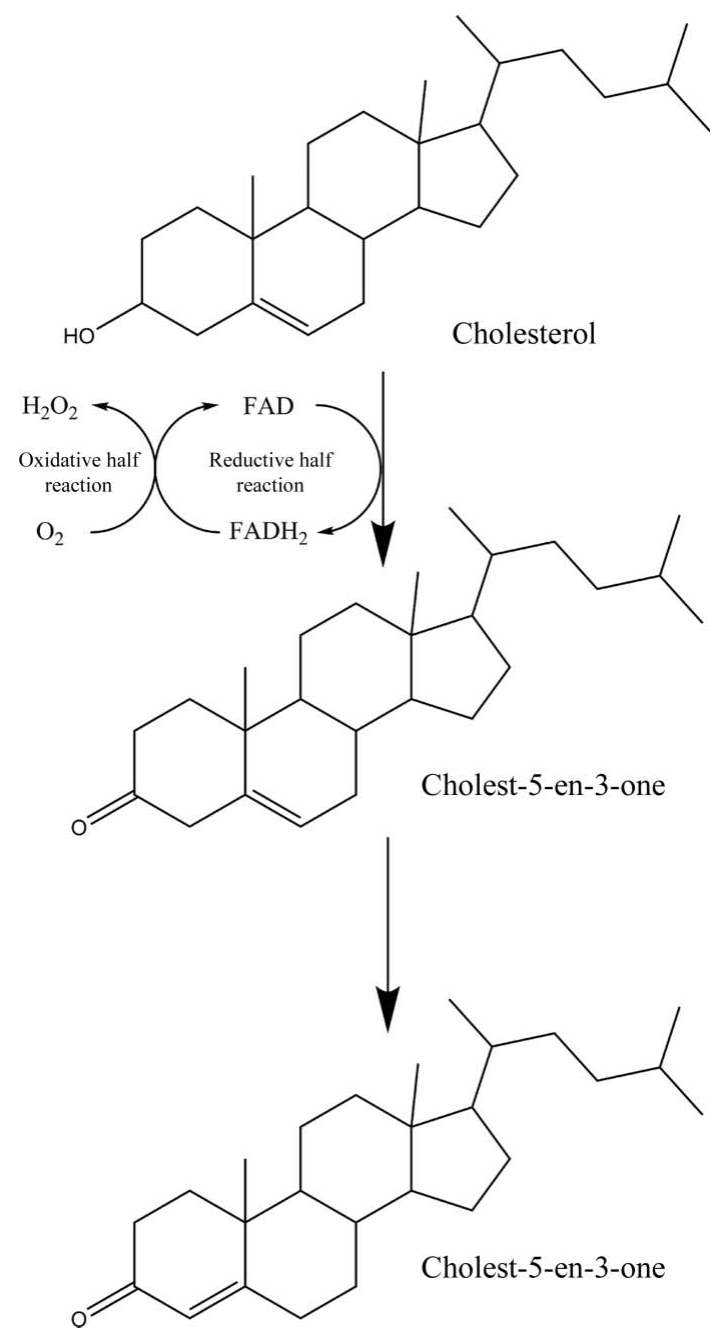

Figure 1

The reaction catalysed by CO. In the reductive half reaction, the substrate hydroxyl proton is abstracted and the hydride is transferred to the FAD cofactor. In the oxidative half reaction the FAD reduces molecular oxygen, producing oxidized FAD and $\mathrm{H}_{2} \mathrm{O}_{2}$. Oxidation of the substrate is followed by isomerization of the double bond from $\mathrm{C} 4$ to $\mathrm{C} 5$. mate, Glu361 (Lyubimov et al., 2006). The correct positioning of the substrate facilitates optimized orbital overlap allowing hydride transfers to occur via a trans-elimination reaction involving an anti-coplanar arrangement of the two $\sigma$ bond orbitals of the substrate. This mechanism further suggests that Glu361 functions as the base for oxidation. However, Glu361 has also been proposed as the base for the isomerization reaction (Kass \& Sampson, 1995, 1998; Sampson \& Kass, 1997; Yamashita et al., 1998). The mechanism of isomerization has been proposed to involve an intramolecular, cis-diaxial transfer of the $\mathrm{C} 4 \beta$ proton to the $\mathrm{C} 6 \beta$ position, forming an enolic intermediate (Sampson \& Kass, 1997). This is supported by the multiple conformations of the Glu361 side chain in crystal structures (Lario et al., 2003; Yue et al., 1999). However, this mechanism implies that, after oxidation and before isomerization, Glu361 must be deprotonated in order that it functions as the base for both steps in the overall enzyme reaction. The exact mechanism by which this would occur is not well understood. At this stage it has not been unequivocally determined whether Glu361 does act as the general base for the abstraction of the substrate hydroxyl proton.

Studies have shown that Asn485 is important for the oxidation reaction by modulating the redox potential of the flavin (Yin et al., 2001). The side chain is found in two conformations, one of which makes an $\mathrm{N}-\mathrm{H} \cdots \pi$ electrostatic interaction with the flavin ring system. Mutagenesis of this residue decreases both $k_{\text {cat }}$ and the redox potential.

Additionally, the side chains of Glu361, Asn 485 and Met122 gate a tunnel which has been proposed to function as a conduit for oxygen access to the reduced flavin within the sequestered enzyme active site (Lario et al., 2003). This channel is gated on the bulk-solvent side by Phe359 (Chen et al., 2008). These residues are found in two conformations in the high-resolution $\mathrm{X}$-ray structures, with their conformations correlated to each other and with an open and closed state of a hydrophobic tunnel extending from the bulk solvent to the active site. It has been proposed that the 'tunnel-open' conformation occurs when the flavin is in the reduced state, facilitating the Asn $485 \mathrm{~N}-\mathrm{H} \cdots \pi$ interaction with the pyrimidine ring of the flavin ring system and thereby stabilizing the reduced FAD. This initial reorientation of the side chain enables further concerted movements of the other tunnel residues, thereby opening the proposed oxygen channel.

Despite the extensive structural work on this enzyme, atomic resolution structures in the presence of a substrate and trapped in the reduced cofactor state have not been characterized. In order to better understand the redox chemistry of the enzyme and the role of the flavin and the surrounding protein residues in modulating redox activity, we have determined atomic resolution crystal structures of the enzyme anaerobically trapped in the reduced state using both 2-propanol and dithionite. Additionally, we have carried out high-level density-functional theory calculations to probe specific interaction energies between the reduced flavin and nearby hydrogen bond-donating groups. These studies provide important insights into the role of specific protein residues in stabilizing the reduced cofactor. 


\section{Materials and methods}

\subsection{Cloning}

The wild-type Streptomyces SA-COO CO gene was amplified from the pCO117 plasmid (Nomura et al., 1995) by polymerase chain reaction (PCR) using the forward and reverse primers 5'-GACTTCATGGCCACTGCACAACAGCATCTG-3' and 3'-GCAGTGCCGCAGCGTGGTGGTGGTGGTGGTGATTCGAACTGA-5', respectively. The reverse primer included codons for six histidine residues. The PCR product was cloned into the $\mathrm{NcoI}$ and HindIII restriction sites of pET-28a-His6-MBP-TEV-AEW (vector kindly provided by Dr Anthony Duff, National Deuteration Facility, ANSTO, Australia). The resulting vector, pCO_P1, was verified by DNA sequencing.

\subsection{Expression and purification}

The structures of the 2-propanol-bound complexes were obtained using the hexahistidine-tagged protein (CO-H6). Expression of CO-H6 was carried out in Escherichia coli BL21 (DE3) cells and purification was performed by nickel-affinity chromatography. Competent E. coli BL21 (DE3) cells were transformed with the pCO_P1 plasmid. Transformants were selected from Luria-Bertani (LB) agar plates supplemented with $50 \mu \mathrm{g} \mathrm{ml}^{-1}$ kanamycin. An overnight inoculant culture was prepared by selecting a single colony from the transformation to inoculate $250 \mathrm{ml} \mathrm{LB}$ containing the above antibiotic. The culture was used to inoculate $312 \times$ YT medium in $6 \times 21$ conical flasks which were shaken at $310 \mathrm{~K}$. When the optical density $(600 \mathrm{~nm})$ reached 0.6 , protein expression was induced by the addition of isopropyl $\beta$-D-1-thiogalactopyranoside (IPTG) to a final concentration of $1 \mathrm{~m} M$ and the cells were incubated for a further $25 \mathrm{~h}$ at $293 \mathrm{~K}$. The cells were harvested by centrifugation at $4500 \mathrm{~g}$ for $45 \mathrm{~min}$ and the cell pellet was flash-frozen in liquid nitrogen at $77 \mathrm{~K}$ until further purification.

The cell pellet was resuspended in lysis buffer (20 m $M$ Tris$\mathrm{HCl} \mathrm{pH} 7.0,500 \mathrm{mM} \mathrm{NaCl}$ ) and lysed with an Emulsiflex C5 high-pressure homogenizer (Avestin). The lysate was clarified by centrifugation at $12600 \mathrm{~g}$ for $30 \mathrm{~min}$ and the supernatant was applied onto a $1 \mathrm{ml}$ Qiagen Ni-NTA column equilibrated with $20 \mathrm{~m} M$ Tris- $\mathrm{HCl} \mathrm{pH} 7.0,500 \mathrm{~m} M \mathrm{NaCl}, 20 \mathrm{~m} M$ imidazole. The protein was eluted with an imidazole gradient (0-500 $\mathrm{m} M$ over 30 column volumes) and fractions were collected. Peak fractions were pooled, dialysed against $20 \mathrm{~m} M$ Tris- $\mathrm{HCl}$ pH 7.0 and concentrated to $6 \mathrm{mg} \mathrm{ml}^{-1}$ as determined by the Bradford assay (Compton \& Jones, 1985) using an Amicon centrifugal filter unit (30 kDa cutoff). Aliquots of the purified protein were snap-frozen in liquid nitrogen and stored at $193 \mathrm{~K}$.

The nonhistidine-tagged version of the protein (CO) was used for structure determination of the dithionite-treated crystals. Competent E. coli BL21 (DE3) pLysS cells were transformed with the pCO117 plasmid (Nomura et al., 1995) and transformants selected from LB agar plates supplemented with $50 \mu \mathrm{g} \mathrm{ml}^{-1}$ ampicillin and $34 \mu \mathrm{g} \mathrm{ml}^{-1}$ chloramphenicol. $50 \mathrm{ml}$ LB containing the above antibiotics was inoculated with a single colony from the transformation and grown overnight at $310 \mathrm{~K}$. This starter was used to inoculate 21 of $2 \times$ YT medium also containing the above antibiotics. The cells were grown at $310 \mathrm{~K}$ until the optical density $(600 \mathrm{~nm})$ reached 0.65 and protein expression was induced by the addition of IPTG to a final concentration of $0.4 \mathrm{~m} M$. The cells were further incubated at $293 \mathrm{~K}$ for $20 \mathrm{~h}$ and harvested by centrifugation at $12000 \mathrm{~g}$ for $30 \mathrm{~min}$. CO was purified according to a previously reported method (Lyubimov et al., 2007). The protein was dialysed into $50 \mathrm{~m} M$ HEPES pH 7 and aliquots were snapfrozen in liquid nitrogen and stored at $193 \mathrm{~K}$.

\subsection{Crystallization and crystal soaks}

Single crystals of both CO-H6 and CO were grown using the conditions previously reported for the nonhistidine-tagged protein (Yue et al., 1999). Crystals were obtained by vapour diffusion using the hanging-drop method in 24-well VDX plates (Hampton Research). The protein $\left(7 \mathrm{mg} \mathrm{ml}^{-1}\right.$ ) was mixed in a $1: 1$ ratio with reservoir solution consisting of $7 \%$ PEG 8000, $100 \mathrm{~m} M$ cacodylate $\mathrm{pH}$ 5.2, $125 \mathrm{mM} \mathrm{MnSO}_{4}$. The drops were immediately seeded with a solution made by crushing previously obtained cholesterol oxidase crystals in stabilizing solution $(12 \%$ PEG $8 \mathrm{~K}, 100 \mathrm{~m} M$ sodium cacodylate $\left.\mathrm{pH} 5.2,125 \mathrm{mM} \mathrm{MnSO}_{4}\right)$. Crystals grew to a volume of $\sim 0.01 \mathrm{~mm}^{3}$.

Single CO crystals were soaked for $3 \mathrm{~h}$ in $1 \mathrm{ml}$ reservoir solution containing varying concentrations of sodium dithionite $(5,10$ and $50 \mathrm{mM})$. The crystals were briefly transferred to a cryoprotectant solution consisting of the soaking solution and $20 \%$ glycerol. Crystals were considered to be reduced when they changed from yellow to colourless. The crystals were placed in CryoLoops (Hampton Research) and flashcooled in liquid nitrogen. Crystals soaked in 10 and $50 \mathrm{mM}$ sodium dithionite were colourless in appearance; however, they exhibited no diffraction. Crystals soaked in $5 \mathrm{mM}$ dithionite almost completely changed from yellow to colourless and maintained high-resolution diffraction.

CO-H6 crystals were placed into a sealed vial containing $1 \mathrm{ml}$ reservoir solution and 20\% 2-propanol. Oxygen was purged from the vial by gently bubbling argon gas through the liquid. 2-Propanol-treated crystals were allowed to soak overnight in the sealed anaerobic vial until they became colourless. A glove bag maintained under an argon atmosphere was used to manipulate the crystals under anaerobic conditions. The crystals were trapped in the colourless state by briefly cryoprotecting them in the soaking solution containing $20 \%$ glycerol and were flash-cooled in liquid nitrogen. Aerobic 2-propanol-soaked crystals were prepared similarly but the soaking solutions were not purged of oxygen, nor were the crystals manipulated in an anaerobic atmosphere; rather, all crystal soaks and manipulations were carried out under aerobic conditions.

To ensure that 2-propanol reduced the protein to the hydroquinone form, absorption spectra were recorded for the oxidized and 2-propanol-reduced CO-H6. Briefly, a cuvette containing $0.5 \mathrm{mg} \mathrm{ml}^{-1} \mathrm{CO}-\mathrm{H} 6$ in $50 \mathrm{~m} M$ Tris- $\mathrm{HCl}$ pH 7.0 was 
Table 1

Data-collection and refinement statistics.

Values in parentheses are for the highest resolution shell.

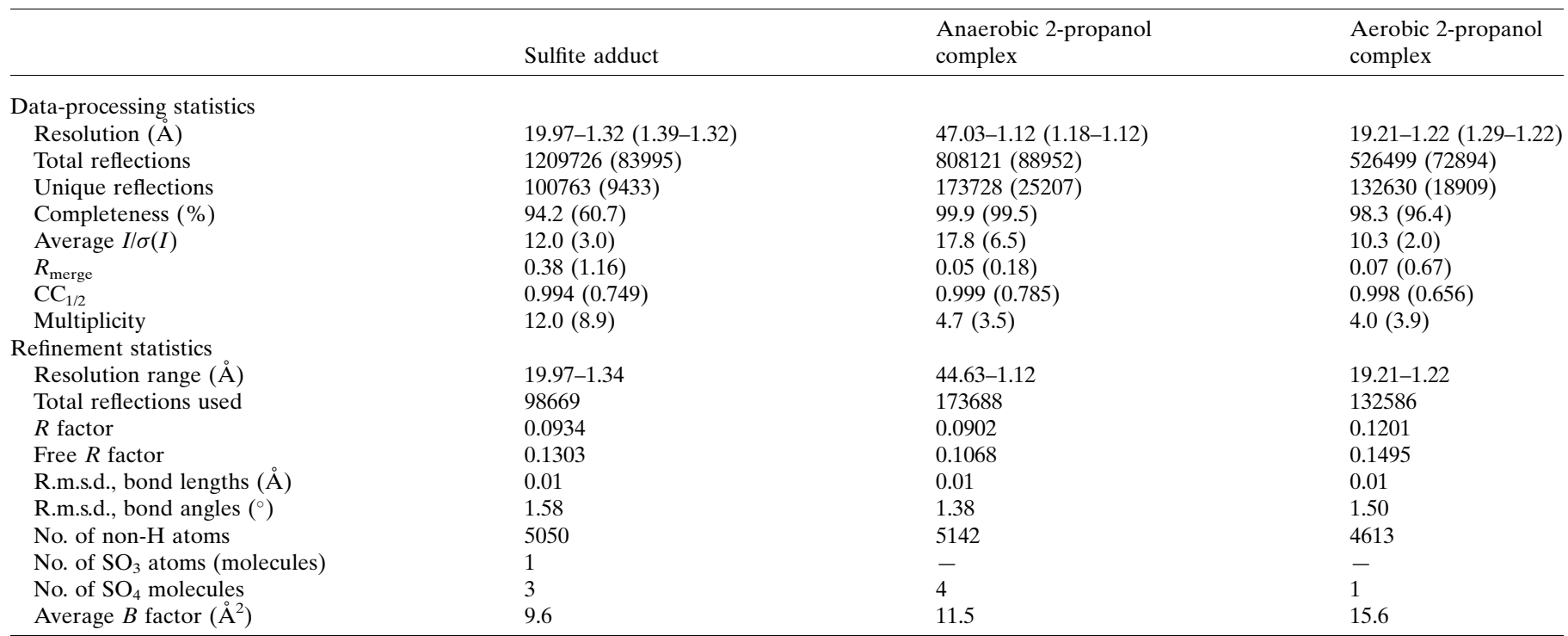

prepared and the absorption spectrum was recorded using a Shimadzu UV-Vis spectrophotometer to obtain the oxidized spectrum. 2-Propanol was then added to the cuvette to a final concentration of $20 \%$, and it was sealed with a rubber septum and purged of oxygen by bubbling argon through the solution for approximately $2 \mathrm{~min}$. The cuvette was placed in an anaerobic glove box overnight to ensure full reduction. The following day, the absorption spectrum of the sample in the cuvette was measured.

\subsection{X-ray data collection, processing and phasing}

X-ray diffraction data sets for all soaked crystals were obtained at the Australian Synchrotron, Melbourne (AS) using an ADSC Quantum 315r CCD detector. Diffraction data for the anaerobic 2-propanol-soaked crystal and for the dithionite-soaked crystal were collected on beamline MX2, while data for the aerobic 2-propanol-soaked crystal were collected on beamline MX1. For all data sets, 360 images were collected using a rotation angle of $1^{\circ}$ per image. Data processing was performed using the $X D S$ software (Kabsch, 2010) and data reduction was carried out using the $C C P 4$ suite of software (Winn et al., 2011).

For all data, crystallographic refinement was carried out starting with a previously determined high-resolution (HR) ligand-free structure of cholesterol oxidase (Lario et al., 2003; PDB entry $1 \mathrm{mxt}$ ). The model was edited to remove the FAD cofactor, water molecules and alternate conformations of residues. The phases were improved through iterative cycles of model building using Coot (Emsley et al., 2010) and the refinement options in PHENIX (Adams et al., 2010) and REFMAC (Winn et al., 2011). The side chains of the oxygentunnel and aromatic triad residues (Tyr444, Tyr107, Phe446, Met122, Phe359 and Asn485) were modelled with partial occupancies in cases where an alternate conformation was not visible in the difference electron density but where negative density appeared and persisted after several cycles of refinement. The data-processing and refinement statistics for each structure are shown in Table 1. The atomic coordinates have been deposited in the Protein Data Bank (accession codes $4 \mathrm{u} 2 \mathrm{l}, 4 \mathrm{u} 2 \mathrm{~s}$ and $4 \mathrm{u} 2 \mathrm{t}$ for the enzyme complexed with sulfite, the enzyme anaerobically reduced with 2-propanol and the enzyme soaked with 2-propanol aerobically, respectively).

\subsection{Computational details}

The geometries and harmonic vibrational frequencies of all structures were obtained at the B3LYP-D3/Def2-TZVPP level of theory (the optimized structures are given in Table $\mathrm{S}^{\mathbf{1}}$; Becke, 1993; Lee et al., 1988; Stephens et al., 1994; Weigend \& Ahlrichs, 2005). Empirical D3 dispersion corrections (Grimme, 2011; Grimme et al., 2011) are included using the Becke-Johnson (Becke \& Johnson, 2005; Johnson \& Becke, 2005, 2006) damping potential as recommended in Grimme et al. (2011) (denoted by the suffix D3). Zero-point vibrational energies and enthalpic temperature corrections have been obtained, within the rigid-rotor harmonic oscillator approximation, from such calculations. All geometry optimizations and frequency calculations were performed using the Gaussian 09 program suite (Frisch et al., 2009). The equilibrium structures in the unconstrained optimizations were verified to have all real harmonic frequencies, whereas the constrained structure is characterized by one imaginary frequency (of $28.324 \mathrm{~cm}^{-1}$ ) that corresponds to the geometry constraint placed on the $\angle \mathrm{N} 10-\mathrm{N} 5-\mathrm{N} 1$ angle.

High-level double-hybrid density-functional theory (DHDFT) calculations (Goerigk \& Grimme, 2014) using the B2GP-PLYP procedure (Karton et al., 2008) were performed

\footnotetext{
${ }^{\mathbf{1}}$ Supporting information has been deposited in the IUCr electronic archive (Reference: DZ5351).
} 
to determine the strength of the hydrogen-bond interactions at the N5 centre. The B2GP-PLYP procedure involves both HF-like exchange and MP2-like correlation in the functional form and thus offers a reliable approach to treat systems that involve weak interactions (Karton et al., 2008; Tarnopolsky et al., 2008). In particular, the B2GP-PLYP functional has been found to yield excellent performance for weak interactions such as hydrogen bonds, dispersion forces and van der Waals

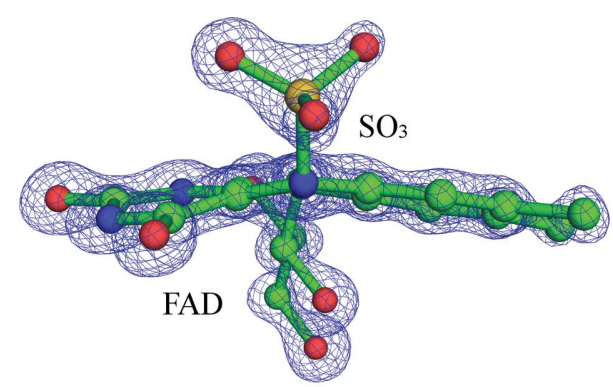

(a)

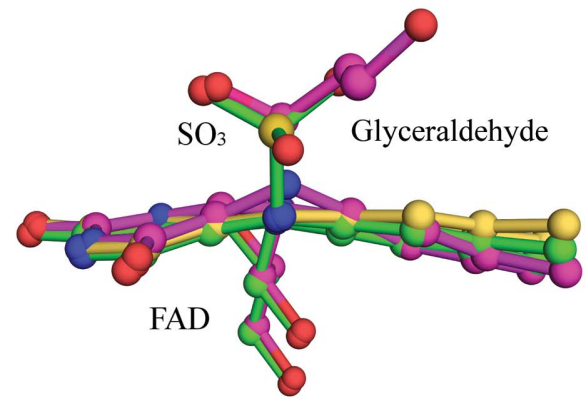

(b)

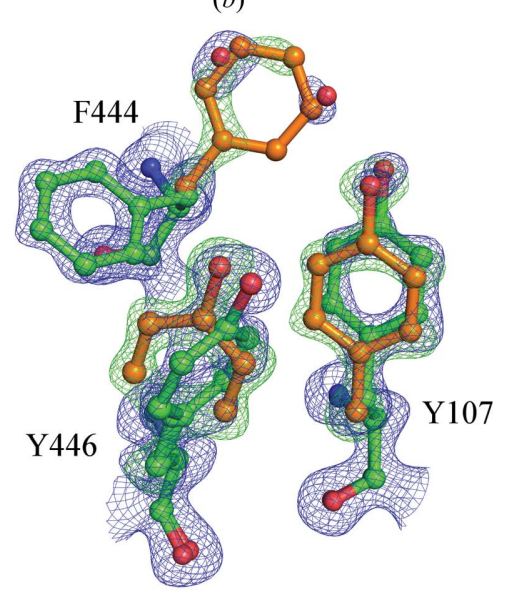

(c)

Figure 2

(a) Electron-density map of the FAD-sulfite adduct. The $2 F_{\mathrm{o}}-F_{\mathrm{c}}$ electron-density map is displayed as a blue mesh (contoured at $2.5 \sigma)$. (b) Superposition of the structures obtained for the sulfite adduct, the glyceraldehyde adduct and the non-adduct-bound cofactor (PDB entry 3b3r for the latter two structures; Lyubimov et al., 2007). The FAD cofactor in the sulfite adduct is displayed in green bonds. The glyceraldehyde-bound adduct is shown in magenta bonds and the nonadduct-bound cofactor is shown in yellow bonds. (c) The aromatic triad residues as observed in the FAD-sulfite adduct structure. Conformation $A$ is shown in green bonds and conformation $B$ is shown in orange bonds. The $2 F_{\mathrm{o}}-F_{\mathrm{c}}$ map is displayed as a blue mesh (contoured at $1.0 \sigma$ ) and the $F_{\mathrm{o}}-F_{\mathrm{c}}$ map is displayed as a green/red mesh (contoured at $\pm 4 \sigma$ ). Density maps were calculated with the atoms of conformation $B$ removed. interactions (Karton et al., 2008). The DHDFT calculations, which inherit the slow basis-set convergence of MP2 to some degree, were carried out in conjunction with the Def2-QZVPP basis set (Weigend \& Ahlrichs, 2005). All DHDFT calculations were performed using the ORCA 3.0.1 program suite, where the resolution of the identity (RI) approximation was used in the MP2 steps (Neese, 2012).

\section{Results}

\subsection{Bound complexes}

3.1.1. Sulfite-FAD adduct structure. The structure of the dithionite-soaked complex crystal was determined to $1.32 \AA$ resolution and revealed a single sulfite molecule as a covalent adduct to the N5 atom of FAD (Fig. 2a). Crystallographic refinement of the covalent adduct resulted in a sulfite occupancy of $0.64 . \mathrm{SO}_{3}$ is an oxidation product of dithionite and is present as a covalent adduct in several other dithionitereduced FAD-containing proteins (Fritz et al., 2002; Tan et al., 2010; Tegoni \& Cambillau, 1994). This electronegative adduct causes a significant displacement of the side chain of Glu361 from previously observed conformations in the HR ligandfree structure (Lario et al., 2003). Furthermore, the repositioning of the side chain of Glu361 restricts the orientation of the side chain of Met122. Previously, these side chains adopted conformations that facilitated the opening and closing of a channel from the exterior of the protein to the internal substrate-binding cavity (Lario et al., 2003). In the adductbound state, Met122 is observed in the open state, while the side chains of Asn 485 and Phe359, which gate the tunnel at the substrate-binding pocket and bulk-solvent sides, respectively, are observed only in the closed conformation.

The covalent bond formed between the sulfite molecule and the N5 atom of FAD causes the flavin N5 atom to adopt a more tetrahedral-like geometry and distorts the dimethylbenzene portion of the isoalloxazine ring away from that observed by Lario et al. (2003) in the HR ligand-free structure. This bending of the cofactor ring system has been reported previously (Lyubimov et al., 2007) when a double-mutant form of the enzyme was soaked in glycerol (Fig. 2b). However, in the current structure a smaller degree of bending along the N5-N10 plane of the isoalloxazine ring was evident relative to that observed in the mutant glycerol co-crystal structure. Furthermore, the structure determined in the presence of glycerol led to two conformations of the isoalloxazine ring, whereas in the current structure only a single conformation was present.

The active site of the enzyme contains a hydrophobic triad consisting of Tyr446, Tyr107 and Phe444. These residues have been observed in two discrete conformations and have been proposed to play a role in substrate-induced bending of the isoalloxazine ring system as the flavin is reduced (Lyubimov et al., 2007). Inspection of these residues in the sulfite-adduct structure revealed similar alternate side-chain conformations to those previously observed in the structure of a double-mutant form of the enzyme where a flavin glyceraldehyde adduct is present (Lyubimov et al., 2007; Fig. 2c). 
3.1.2. 2-Propanol-bound complex structures. 2-Propanol is a small alcohol substrate and has previously been reported to reduce FAD slowly (Pollegioni et al., 1999). Structures of the enzyme in complex with 2-propanol were determined to 1.12 and $1.22 \AA$ resolution for crystals soaked in solutions containing the substrate under anaerobic and aerobic conditions, respectively. In the anaerobic case the crystals bleached, suggesting that the enzyme was trapped in the reduced state, whereas in the aerobic 2-propanol case the crystals remained yellow in colour, suggesting that the enzyme had reverted back to the oxidized state. The absorption spectra of the

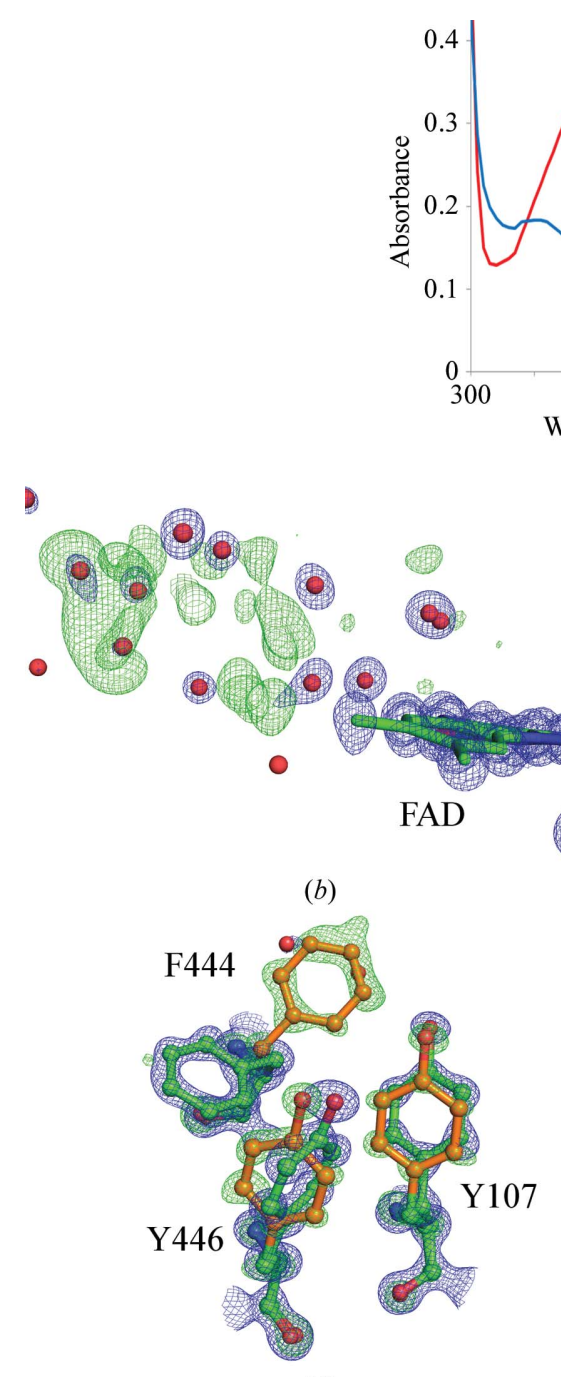

(d)

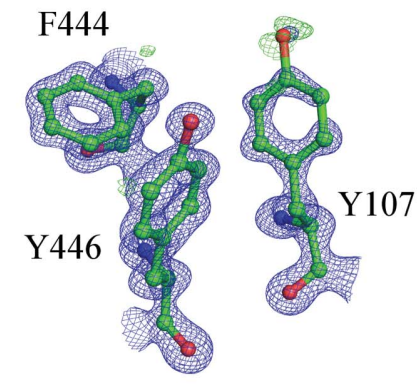

(e)

\section{Figure 3}

(a) Absorption spectra of the oxidized (red curve) and reduced (blue curve) states of the cholesterol oxidase enzyme. $(b, c)$ Residual difference density in the binding pocket of the reduced and oxidized structures, respectively. The $2 F_{\mathrm{o}}-F_{\mathrm{c}}$ maps for $(b)$ and $(c)$ are displayed as a blue mesh (contoured at $1.5 \sigma$ ) and the $F_{\mathrm{o}}-F_{\mathrm{c}}$ maps are displayed as a green/red mesh (contoured at $\pm 3.5 \sigma$ ). (d) Electron-density maps for the aromatic triad amino-acid residues of the reduced anaerobic 2-propanol structure. The $2 F_{\mathrm{o}}-F_{\mathrm{c}}$ map is displayed as a blue mesh (contoured at $1.5 \sigma$ ) and the $F_{\mathrm{o}}-F_{\mathrm{c}}$ map is displayed as a green/red mesh (contoured at $\pm 4 \sigma$ ). (e) Electron-density maps for the aromatic triad amino-acid residues of the aerobic 2-propanol structure. The $2 F_{\mathrm{o}}-F_{\mathrm{c}}$ map (contoured at $1.5 \sigma$ ) is displayed as a blue mesh and the $F_{\mathrm{o}}-F_{\mathrm{c}}$ map (contoured at $\pm 3.0 \sigma$ ) is displayed as a green/red mesh. Conformation $A$ of the triad residues is shown in green bonds and conformation $B$ of the residues is shown in orange bonds. Maps were calculated with the atoms of conformation $B$ removed for $(d)$. oxidized and anaerobic 2-propanol-soaked enzyme in solution showed that $\mathrm{CO}$ is reduced to the hydroquinone form by 2-propanol (Fig. 3a). The high resolution of the data enables near-atomicity for many of the atoms in the structure.

Difference electron density was apparent in the substratebinding pocket for each of the 2-propanol structures (Figs. $3 b$ and $3 c$ ), suggesting the presence of 2-propanol. However, modelling of 2-propanol into the density and refinement did not result in convincing $2 F_{\mathrm{o}}-F_{\mathrm{c}}$ density; therefore, the substrate molecules were not included in the final structures. It is likely that the small size of the substrate relative to cholesterol, the natural substrate of the enzyme, results in multiple disordered binding modes and thus poorly defined difference electron density.

The residues comprising the aromatic triad (Tyr107, Tyr446 and Phe444) adopt two conformations (Fig. $3 d$ ) in the anaerobic structure, whereas they are only found in a single conformation in the aerobic structure (Fig. 3e). In the former case, the alternate side-chain conformation $(B)$ was modelled into well defined positive difference density but refined only to low occupancy $(0.28$, 0.24 and 0.24 , respectively), resulting in poor $2 F_{\mathrm{o}}-F_{\mathrm{c}}$ density for this region. However, the well defined difference density and the low temperature factors for these residues (9.63, 7.07 and $11.3 \AA^{2}$ ) strongly suggest the presence of the alternate conformations despite poor $2 F_{\mathrm{o}}-F_{\mathrm{c}}$ density.

During the refinement of the anaerobic 2-propanol complex structure, a positive difference electron-density peak became apparent at the $>3.5 \sigma$ contour level positioned $1.03 \AA$ from the isoalloxazine $\mathrm{N} 5$ atom in a tetrahedral geometry $(\angle \mathrm{C} 5 \mathrm{X}-\mathrm{N} 5-\mathrm{HN} 5$ angle of $\sim 111^{\circ}$ ), suggesting that a hydride transfer had occurred from the 2-propanol substrate (Fig. 4a). Modelling of an $\mathrm{H}$ atom at full occupancy into this difference density removed the residual difference density. Interestingly, a similar difference electrondensity peak was not present in the maps of the aerobic 2-propanol complex even at very low difference map contour levels (Fig. 4b).

Hydride transfer to N5 of the flavin induced no further significant bending of the isoalloxazine moiety from that in the oxidized structure. A hydrogen bond $(2.36 \AA)$ between the flavin N5 atom and the backbone amide $\mathrm{H}$ atom 
of Gly120 completes the tetrahedral geometry about N5. Theoretical calculations have been performed on free flavins and flavins within protein structures (Cavelier \& Amzel, 2001; Walsh \& Miller, 2003; Zheng \& Ornstein, 1996); however, no experimental evidence for the position of the $\mathrm{H}$ atom has been obtained owing to the limitations of X-ray crystallography for
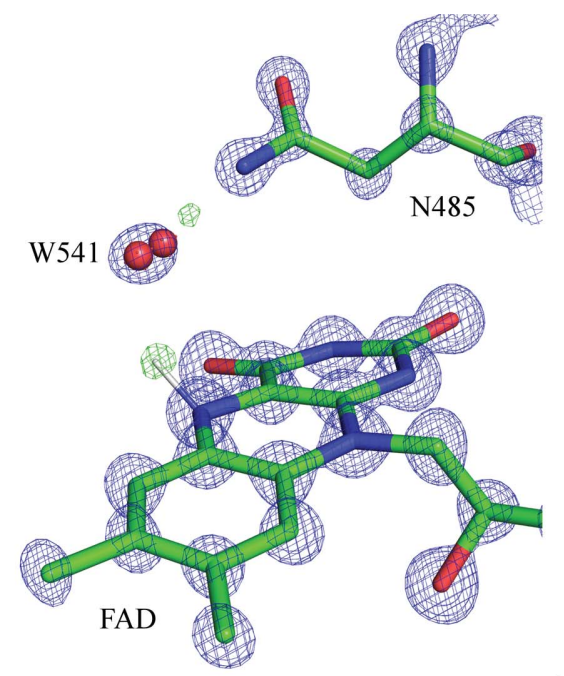

(a)
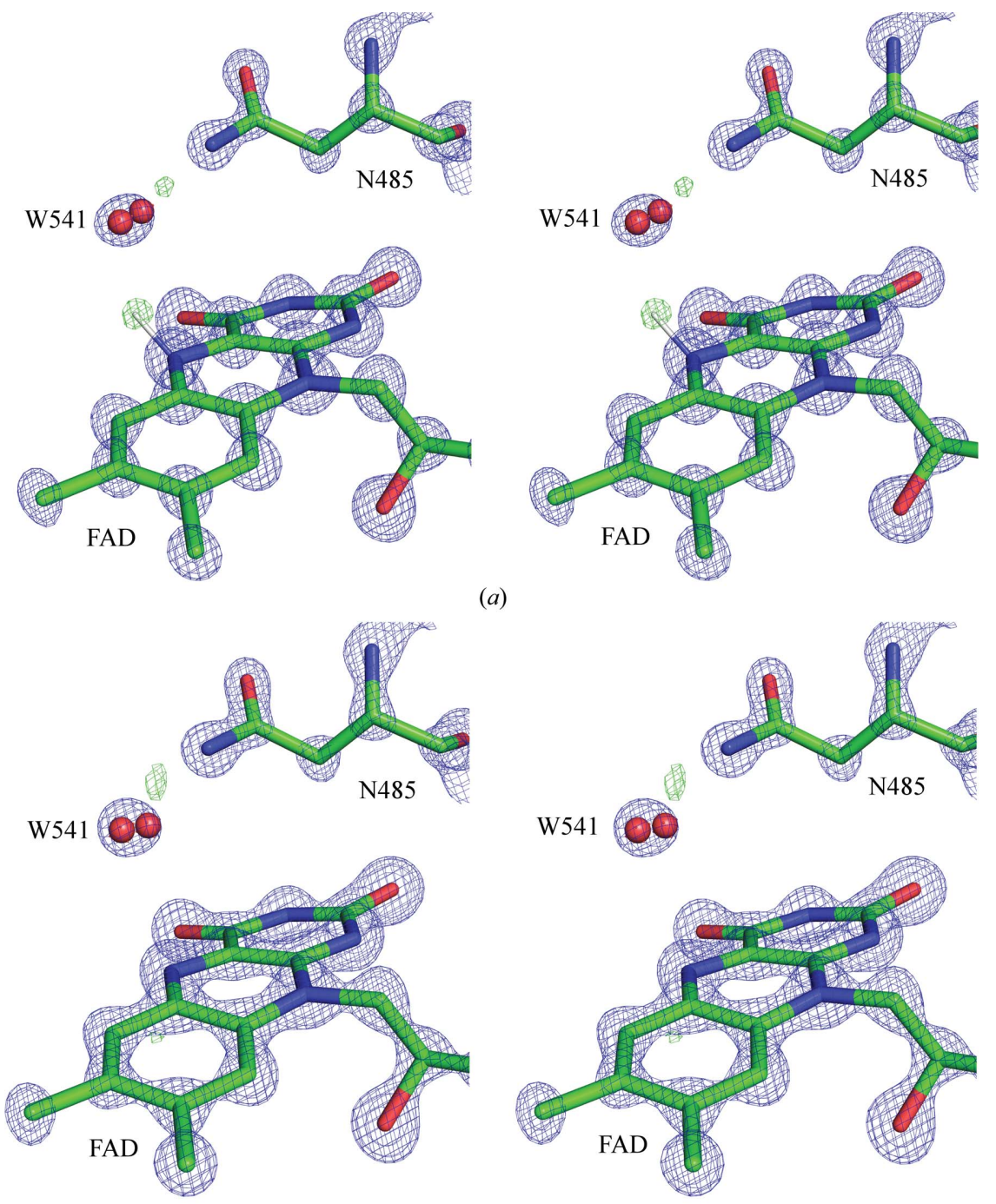

(b)

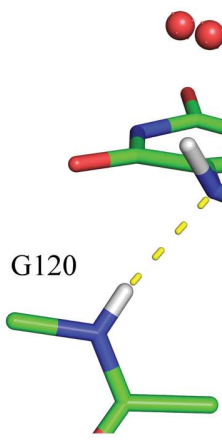

W541

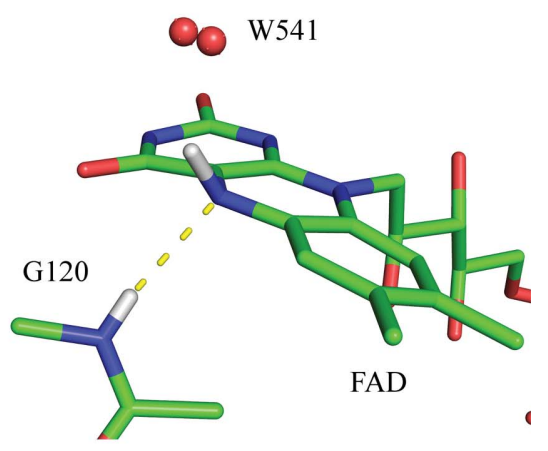

(c)

Figure 4

Wall-eyed stereoviews of the FAD cofactor in the anaerobic $(a)$ and aerobic $(b)$ 2-propanolsoaked structures. The $2 F_{\mathrm{o}}-F_{\mathrm{c}}$ electron-density map is shown as a blue mesh (contoured at $2.5 \sigma$ ) and the $F_{\mathrm{o}}-F_{\mathrm{c}}$ density map is shown as a green/red mesh (contoured at $\left.\pm 3.5 \sigma\right)$. (c) The hydrogen bond formed between the Gly120 amide $\mathrm{H}$ atom and the flavin N5 atom. locating $\mathrm{H}$ atoms except at atomic resolution (Elias et al., 2013). High-level DHDFT calculations using the B2GP-PLYP procedure (Karton et al., 2008) were performed in order to probe the strength of the hydrogen bond formed between Gly120 (Fig. 4c) and the reduced isoalloxazine system $\left(\mathrm{FADH}^{-}\right)$and to assess the possible implications of this hydrogen-bonding interaction on the $\mathrm{FADH}^{-}$structure. For reasons of computational efficiency, the ribitol-ADP side chain on the flavin and the Gly120 residue were modelled by a methyl group and a dimethylamine moiety, respectively. Our model systems are shown in Fig. 5, and Table 2 gives the hydrogen-bond strengths and selected bond distances and angles for these structures. It is instructive to begin with the structure of the reduced isoalloxazine without any hydrogen-bonding interactions (Fig. 5a). This structure is characterized by a bending angle of the isoalloxazine of $158.3^{\circ}$ and a shallow angle for the position of the transferred hydride of $165.5^{\circ}$ (Table 2). Adding a dimethylamine to the calculations, to mimic the $R_{2} \mathrm{~N} 1-\mathrm{H} 1$ functional group of Gly120, results in a strong hydrogen-bond interaction of $7.47 \mathrm{kcal} \mathrm{mol}^{-1}$ between the negatively charged isoalloxazine $\left(\mathrm{FADH}^{-}\right)$ and the dimethylamine hydrogen-bond donor (Fig. $5 b$ and Table 2). The effectiveness of this interaction is also evident by a relatively short hydrogen-bond distance $\left(\mathrm{FADH}^{-}\right.$...

$\mathrm{H}-\mathrm{N}$ ) of $2.252 \AA$. In the fully optimized structure the dimethylamine is situated directly above the N5 atom such that the $\angle \mathrm{N} 10-\mathrm{N} 5-\mathrm{H} 1$ angle is $85.8^{\circ}$ (Fig. $5 b$ ). This situation allows an effective overlap between the lone pair on the N5 atom and the N1-H1 bond of the dimethylamine. Constraining the $\angle \mathrm{N} 10-\mathrm{N} 5-\mathrm{N} 1$ angle in our model to be $125.5^{\circ}$ allows a less effective overlap between the lone pair on $\mathrm{N} 5$ and the H1-N1 bond (Fig. 5c). Consequently, the strength of the hydrogen bond is reduced to $5.45 \mathrm{kcal} \mathrm{mol}^{-1}$ and the hydrogen-bond distance is slightly elongated to $2.432 \AA$ (Table 2). Constraining the $\mathrm{H} 1-\mathrm{N} 1$ bond to be at a similar position as in the crystal structure has two important structural consequences.

(i) The isoalloxazine ring system becomes less bent. In particular, the bending angle $(\alpha)$ increases from a value of $157.2^{\circ}$ (in the fully optimized model; Fig. $5 b$ ) to a value of $164.9^{\circ}$ (in the constrained model; Fig. 5c).

(ii) The $\mathrm{H}$ atom attached to $\mathrm{N} 5$ shifts further away from the plane of the central 
Table 2

Selected bond lengths and angles of the $\mathrm{FADH}^{-}$and calculated strength of the $\mathrm{FADH}^{-} \ldots \mathrm{HNMe}_{2}$ hydrogen bond $\left(\Delta H_{298}\right)$.

The geometries are optimized at the B3LYP-D3/TZVPP level of theory and the hydrogen-bond energy is calculated at the B2GP-PLYP/QZVPP level of theory. Atom numbers are shown in Fig. 5(b).

\begin{tabular}{llllll}
\hline & $\begin{array}{l}\Delta H_{298} \\
\left(\mathrm{kcal} \mathrm{mol}^{-1}\right)\end{array}$ & $\alpha \dagger$ & $\begin{array}{l}\angle \mathrm{N} 10-\mathrm{N} 5 \\
-\mathrm{H} 5\left(^{\circ}\right)\end{array}$ & $\begin{array}{l}\angle \mathrm{N} 10-\mathrm{N} 5 \\
-\mathrm{H} 1\left(^{\circ}\right)\end{array}$ & $\begin{array}{l}\mathrm{N} 5 \cdots \mathrm{H} 1 \\
\left(^{\circ}\right)\end{array}$ \\
\hline $\mathrm{FADH}^{-} \ddagger$ & & 158.3 & 165.6 & & \\
FADH $^{-} \cdots \mathrm{HNMe}_{2} \S$ & 7.47 & 157.2 & 164.0 & 85.8 & 2.252 \\
FADH $^{-} \cdots \mathrm{HNMe}_{2} \uparrow$ & 5.45 & 164.9 & 154.3 & 124.6 & 2.432 \\
FADH $^{-} \cdots \mathrm{Gly}^{\circ}+\dagger$ & & 175.8 & 129.0 & 127.4 & 2.374 \\
\hline
\end{tabular}

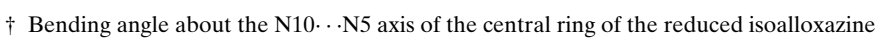
(see Fig. 5a). ‡ Structure shown in Fig. 5(a). \& Structure shown in Fig. 5(b). - The $\angle \mathrm{N} 1-\mathrm{N} 5-\mathrm{N} 1$ angle is constrained to the value in the crystal structure (see Fig. $5 c$ ). $\quad \dagger$ Crystal structure.

ring; in particular, the $\angle \mathrm{N} 10-\mathrm{N} 5-\mathrm{H} 5$ angle decreases from a value of $164.0^{\circ}$ (in the fully optimized model; Fig. 5b) to a value of $154.3^{\circ}$ (in the constrained model; Fig. $5 c$ ).

\section{Discussion}

In this study, structures of cholesterol oxidase were determined from crystals which were trapped in the reduced state using either an alcohol substrate or dithionite, a strong reducing agent that is commonly used to study the redox chemistry of flavoenzymes (Ghisla \& Massey, 1986).

Crystals of the enzyme with an N5-sulfite adduct were prepared under aerobic conditions and the crystals bleached from yellow to colourless. Additionally, crystals of the enzyme soaked in an alcohol substrate were prepared under aerobic conditions and anaerobic conditions. Only in the case of the anaerobic soaking conditions did the crystals undergo a colour change from yellow to colourless and remain colourless, indicative of a trapped reduced form of the enzyme. In this case, the absence of $\mathrm{O}_{2}$ prevented the enzyme from undergoing the oxidative half reaction. In the dithionite-soaked crystals a trapped covalent flavin-sulfite adduct was observed. In contrast, in the presence of 2-propanol no covalent adduct was apparent for either the aerobic or anaerobic treated crystals. Comparisons of active-site residues between the two 2-propanol-treated crystals show differences in side-chain conformations and in the structure of the isoalloxazine ring system of the cofactor which provide insights into the mechanism by which the protein accommodates the reduced state of the cofactor.

The covalent sulfite-adduct structure revealed two conformations of the aromatic triad side chains and bending of the isoalloxazine ring system of the FAD. These conformational changes have previously been observed for crystals of an enzyme double mutant (H447Q/E361Q) which were soaked in glycerol; in this case a covalent adduct was observed between the FAD N5 atom and the reduced glycerol product, glyceraldehyde (Lyubimov et al., 2007). Additionally, a structure of a CO from Brevibacterium sterolicum (Li et al., 1993) in its reduced state with a noncovalently bound steroid molecule also showed movements of the side chains within the aromatic triad compared with the unbound structure. Our studies using dithionite further support the proposal that bending of the flavin and movement of the aromatic triad residues is linked to ligand binding and the redox state of the cofactor (Lyubimov et al., 2007). In the previous study of the glyceraldehyde

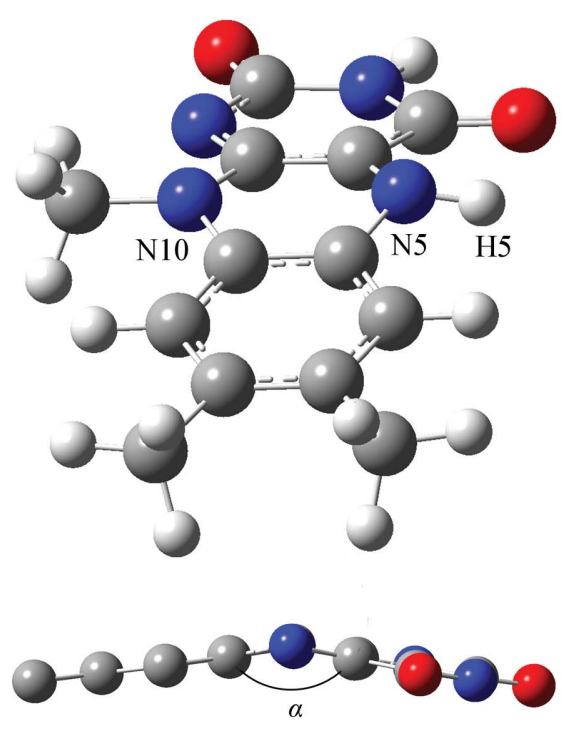

(a)

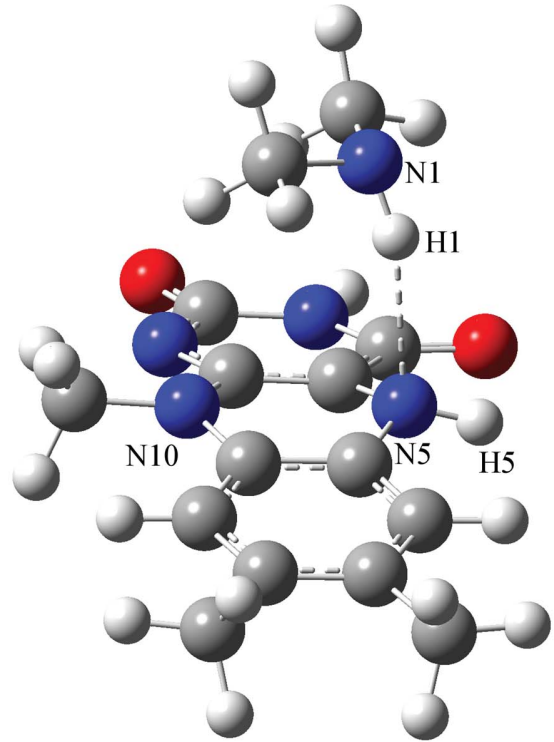

(b)

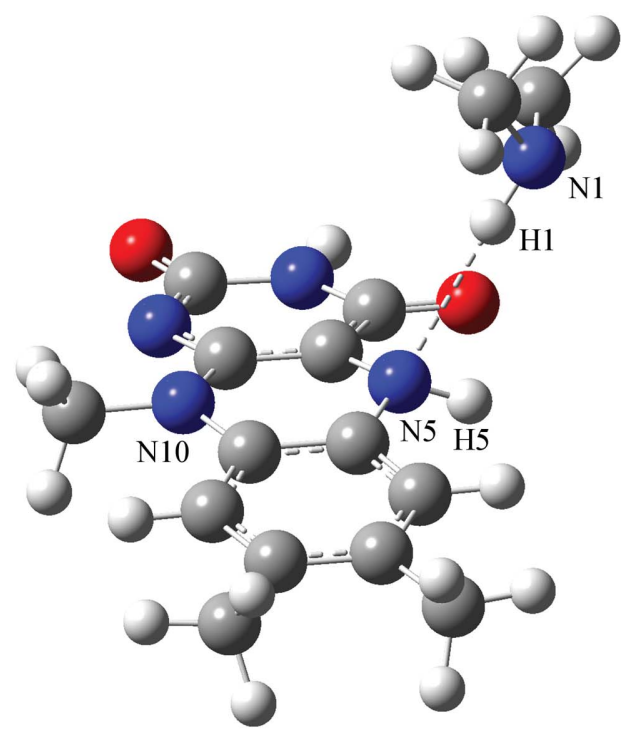

(c)

Figure 5

Optimized structures obtained at the B3LYP-D3/TZVPP level of theory of $(a)$ the reduced isoalloxazine system and $(b, c)$ the reduced isoalloxazine with a hydrogen-bonding interaction with a dimethylamine moiety at N5. The structures in $(a)$ and $(b)$ are fully optimized, whereas in that in $(c)$ the value of the angle between the $\mathrm{N} 10 \cdots \mathrm{N} 5$ axis of the central ring and the dimethylamine $\mathrm{N}$ atom (N1) is kept fixed at the value in the crystal structure $\left(125.5^{\circ}\right)$. The bending angle $(\alpha)$ about the N10 $\cdots \mathrm{N} 5$ axis of the central ring is shown in $(a)$; the lower panel also shows $\alpha$ in a side view of FADH ${ }^{-}$(in which the $\mathrm{H}$ atoms have been omitted for clarity). The atom numbers used in Table 2 and in the text are shown in (b). Atomic colour scheme: $\mathrm{H}$, white; $\mathrm{C}$, grey; $\mathrm{N}$, blue; $\mathrm{O}$, red. 
adduct structure, the high resolution of the structures enabled two conformations of the isoalloxazine ring to be observed: one conformation adopts the typical butterfly twist, while the second conformation exhibits a greater degree of bending of the dimethylbenzene moiety, presumably owing to steric pressure exerted by the aromatic triad. The different conformations of the flavin were correlated with the amount of covalent adduct that was present in the model. In the present sulfite-adduct structure, despite the relatively high occupancy of the adduct (0.63), the density maps did not reveal as significant a distortion in the isoalloxazine ring conformation as was observed in the double-mutant/glycerol structure. This difference may be owing to the lower resolution of the structure $(1.3 \AA)$ relative to the double-mutant structure $(0.98 \AA)$. Furthermore, the degree of bending seen in the FAD/sulfite adduct was less than that observed in the glyceraldehyde-adduct structure This apparent lesser degree of bending of the flavin ring may also be a consequence of the limited resolution of the sulfite-adduct structure, which only provides a view of the average of the covalent flavin-adduct and the unbound states. Therefore, although we cannot establish the exact extent of flavin bending in the sulfite-bound structure at the current resolution, the multiple conformations of the aromatic triad provide strong supportive evidence that the presence of a ligand covalently bound to the flavin cofactor invokes changes to the active site of the enzyme that include conformational changes to the flavin moiety.

Substrate oxidation by the enzyme is not expected to involve a covalent flavin adduct. In the case of the glyceraldehyde-adduct structure, adduct formation is likely to have occurred after substrate oxidation of the primary alcohol group of glycerol to form glyceraldehyde, which reacts further, resulting in a covalent complex with FAD. In contrast, 2-propanol is a secondary alcohol substrate that mimics the secondary alcohol of cholesterol. Oxidation of a secondary alcohol results in the formation of a ketone, which cannot undergo further reaction with FAD to form a covalent adduct. We therefore expect the 2-propanol-bound forms to more accurately represent the true substrate-reduced state of the enzyme.

Interestingly, the anaerobic 2-propanol-bound structure showed a positive difference density peak positioned within bonding distance of the flavin N5 atom. The position of this density, and the bleached appearance of the anaerobic crystals, suggests that the enzyme has undergone a hydride transfer from 2-propanol to N5 of the flavin. This state also shows the presence of multiple conformations of the aromatic triad residues which were not observed in the aerobic 2-propanol structure. The high occupancy $(>0.5)$ of the bound steroid substrate ( $\mathrm{Li}$ et al., 1993), the glyceraldehyde adduct (Lyubimov et al., 2007) and our current sulfite adduct all correlate with a high occupancy of the alternate conformation of the aromatic triad. The 2-propanol-bound structures both exhibit poorly occupied ligand in the binding site. This suggests that the alternate aromatic triad conformations are owing to two factors: steric pressure induced by a highly occupied ligand and reduction of the cofactor without bending of the flavin ring system. The second conformation of the aromatic triad orients the more positively charged periphery of the Tyr446 ring (Chakrabarti \& Bhattacharyya, 2007; Hunter, 1993; Hunter \& Sanders, 1990; McGaughey et al., 1998) towards the dimethylbenzene moiety of the cofactor. This interaction may help to stabilize the increased electronegative state of the reduced cofactor.

FAD can exist in oxidized, semiquinone or hydroquinone states depending on the mechanism and the redox state of the cofactor. Structures of these flavin states have been investigated by theoretical calculations and suggest that the isoalloxazine ring system of free oxidized flavin is planar, while that of the reduced flavin is bent about the N5-N10 axis to form a 'butterfly' conformation (Hall et al., 1987; Walsh \& Miller, 2003; Zheng \& Ornstein, 1996). Zheng \& Ornstein (1996) also suggested, however, that the anionic form of flavin may be planar. Crystal structures of flavoproteins have also revealed planar ring conformations for the oxidized state (Waksman et al., 1994) as well as bent conformations for the reduced state [Porter \& Voet, 1978; Singh et al., 2014; PDB entries 3swo and 3sf6 (Seattle Structural Genomics Center for Infectious Disease, unpublished work)]. The ring conformation of the flavin is highly influenced by the local protein environment, which modulates the flavin both structurally (largely through hydrogen-bond interactions) and through redox activity (Li \& Fu, 2008; Røhr et al., 2010; Rotello, 1999). Examples of flavoproteins have been reported in which the flavin changes from a planar oxidized state to a bent reduced state (Lennon et al., 1999; Senda et al., 2007; Waksman et al., 1994). In contrast, other reports have shown reduced flavins with little or no change in the ring conformation between the oxidized and reduced states (Dobbek et al., 2002; Faust et al., 2007; Li et al., 1993; Sukumar et al., 2004; van den Hemel et al., 2006; van Straaten et al., 2012).

Comparisons of the oxidized and 2-propanol-reduced structures of $\mathrm{CO}$ indicate no significant change in the isoalloxazine ring conformations. In contrast, the formation of a covalent adduct to N5 as seen in the sulfite-bound structure does result in an increase in the extent of ring bending. These finding are in further agreement with other sulfite-bound flavins reported for alditol oxidase, adenosine-5'-phosphate reductase and pyranose oxidase (Forneris et al., 2008; Schiffer et al., 2006; Tan et al., 2010). In the case of flavocytochrome B2, the sulfite adduct only moves the N5 atom to lie out of the plane of the normally planar isoalloxazine ring (Mowat et al., 2000, 2004).

Density-functional theory calculations on flavin derivatives showed that increasing the imposed bend angle of the flavin increased the relative stability of the reduced hydroquinone flavin compared with the semiquinone form (Walsh \& Miller, 2003). Furthermore, these studies showed that the conformational energy of the FAD was correlated to the midpoint reduction potential such that a bent flavin conformation is a better electron acceptor while a planar conformation is a better electron donor.

Our results for the covalent adduct and the anaerobic 2-propanol-reduced state of the enzyme provide experimental 
validation that the formation of the covalent adduct, and not the reduction of the flavin per se, causes the isoalloxazine ring system to bend further from its oxidized state. Indeed, the protein environment around the flavin stabilizes the isoalloxazine ring away from planarity, thereby priming the cofactor for reduction. Further bending is mediated only through formation of a covalent adduct.

We suggest that the difference density peak near the flavin N5 atom of the anaerobic 2-propanol-reduced structure indicates the presence of an $\mathrm{H}$ atom. This peak is not present in the aerobic 2-propanol structure, where the crystals have not undergone bleaching and hence the flavin is not reduced. The difference density peak is positioned $1.03 \AA$ from the flavin $\mathrm{N} 5$ atom, consistent with an $\mathrm{N}-\mathrm{H}$ bond. While a number of crystallographic structures of reduced flavins have been reported at below $1.5 \AA$ resolution (Gustafsson et al., 2007; Johansson et al., 2010; Khan et al., 2005; Røhr et al., 2010; Sedláček et al., 2014; Sukumar et al., 2004), the resolution of these structures is still insufficient to establish the location of the hydride bound to the flavin. The backbone amide $\mathrm{NH}$ group of Gly120 is positioned below the isoalloxazine ring system and functions as hydrogen-bond donor to the flavin N5 atom. A similar hydrogen-bond interaction with the hydrogenbond donor group originating from a backbone amide below the isoalloxazine ring has been observed in several other flavoenzymes, including glucose oxidase from Penicillium amagasakiense (Wohlfahrt et al., 1999), S-mandelate dehydrogenase (Sukumar et al., 2004), D-amino-acid oxidase (Mattevi et al., 1996) and $\mathrm{NAD}(\mathrm{P}) \mathrm{H}$ :acceptor oxidoreductase (FerB) from Paracoccus denitrificans (Sedláček et al., 2014).

Intriguingly, the structure of a mutant form of Shewanella yellow enzyme 1 has been reported in the Protein Data Bank

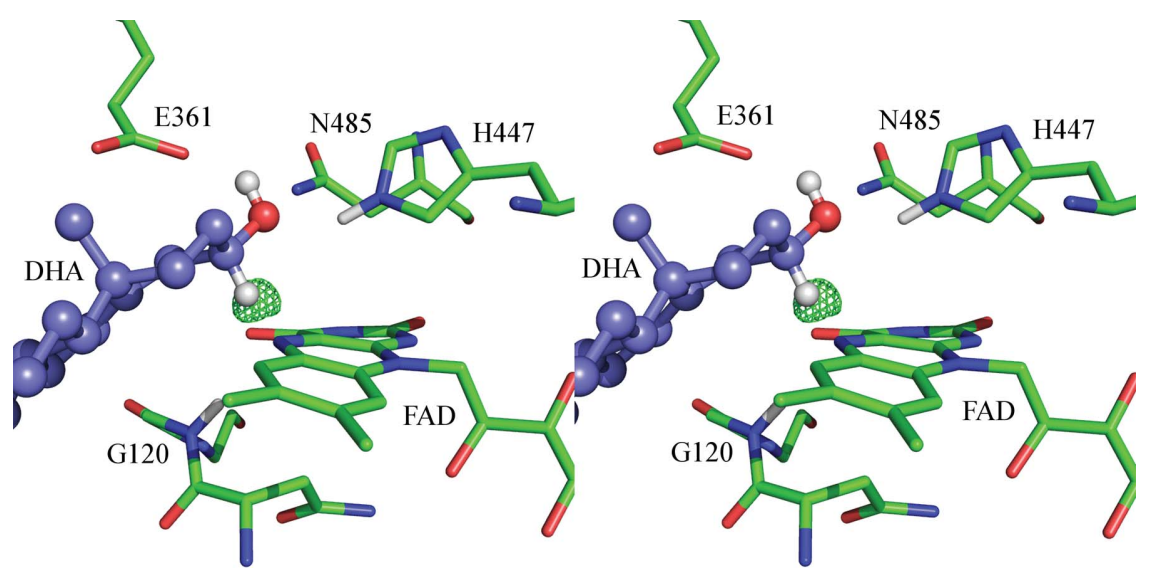

Figure 6

Wall-eyed stereoview of the proposed binding model of the steroid substrate in the active site of CO. The positions of all residues are those observed in the anaerobic reduced structure. Dehydroisoandrosterone (DHA; shown as a blue ball-and-stick representation) has been modelled into the protein structure (green bonds) by positioning the substrate hydroxyl $\mathrm{O}$ atom at the location of the conserved water molecule (Wat541). The molecule was positioned to enable a hydrogen-bond interaction from the substrate $\mathrm{O}$ atom and NE2-HE2 of His447 as well as a hydrogen-bond interaction between the substrate $\mathrm{H}$ atom and the side chain of Glu361. The observed $F_{\mathrm{o}}-F_{\mathrm{c}}$ electron density $(3.5 \sigma)$ for the transferred hydride in the reduced structure is shown as a green mesh to compare the relative positioning of the substrate $\mathrm{C}-\mathrm{H}$ in the model with the hydride location in the reduced structure.
(Berman et al., 2000) in oxidized and reduced states at 1.0 and $0.98 \AA$ resolution, respectively (PDB entries 4aws and 4awt, respectively; J. Elegheert, E. Pauwels, G. Wille, A. Brige \& S. N. Savvides, unpublished work). Inspection of the electrondensity maps for these structures reveals the presence of a difference density peak $(3 \sigma) 1.07 \AA$ from the flavin N5 atom only in the reduced enzyme structure, suggesting that a hydride transfer had occurred. This difference peak is also tetrahedrally oriented about $\mathrm{N} 5$. The amide $\mathrm{NH}$ group of Thr 26 forms a hydrogen bond to N5 from the opposite side of the isoalloxazine ring system.

The conservation of a hydrogen bond between the mainchain amide NH group and the flavin N5 atom in the structures of cholesterol oxidase, D-amino-acid oxidase, glucose oxidase choline oxidase, $S$-mandelate dehydrogenase, NAD(P)H:acceptor oxidoreductase and Shewanella yellow enzyme suggests an important role for this interaction in stabilizing the N5 atom during catalysis. Indeed, our densityfunctional theory calculations suggest that this hydrogen bond influences the position of the hydride on the N5 atom. In the fully optimized structure, the strength of the hydrogen bond formed between the dimethylamine and the negatively charged isoalloxazine system is $7.47 \mathrm{kcal} \mathrm{mol}^{-1}$ (Table 2) and the $\mathrm{H} 1-\mathrm{N} 1$ bond of the dimethylamine moiety is situated irectly above the N5 centre. Constraining the $\angle \mathrm{N} 10-\mathrm{N} 5-$ 1 angle in our model to be the same as that we observe in the thal structure results in a less effective overlap between the pair on $\mathrm{N} 5$ and the $\mathrm{H} 1-\mathrm{N} 1$ bond. Consequently, the po hydrogen bond is reduced and the hydrogendistance is slightly elongated. Constraining the $\mathrm{H} 1-\mathrm{N} 1$ bond also has two important structural consequences which are consistent with what we observe in our reduced crystal structure: the isoalloxazine ring system becomes less bent and the $\mathrm{H}$ atom attached to N5 shifts further away from the plane of the central ring compared with the free and fully optimized system. In the fully optimized model (Fig. 5b) the hydrogen-bond interaction between the dimethylamine and the flavin $\mathrm{N} 5$ atom has little effect on the structure of the isoalloxazine system relative to the free isoalloxazine (Table 2). Therefore, the observed structural changes to the isoalloxazine ring can be attributed to the position of the hydrogen-bond donor (Gly120), which results in a movement of the lone pair of electrons at the N5 centre from lying directly above the $\mathrm{N}$ atom in the fully optimized model (Fig. $5 b$ ) towards the N5 $\cdots$ H1 axis in the constrained model (Fig. $5 c)$.

A conserved water molecule is observed at a hydrogen-bond distance from the flavin N5 atom and from NE2 of His447. Lario and coworkers have proposed that this water molecule mimics the position of the substrate hydroxyl group prior to hydride 
transfer. Furthermore, a hydrogen-bond interaction between HE2 of His447 and the lone-pair electrons on the substrate O atom orients the hydroxyl $\mathrm{H}$ atom towards the proposed base, Glu361. This results in an energetically favourable trans arrangement between the substrate hydroxyl $\mathrm{H}$ atom and the hydride (Lario et al., 2003). Interestingly, when dehydroisoandrosterone is modelled into the anaerobic reduced structure with the above considerations, the substrate $\mathrm{C} 3$ atom lies $2.5 \AA$ away from the flavin N5 atom and in line with the position of the hydride, forming an angle of $128^{\circ}$ with the N5N10 atoms (Fig. 6). Rigid positioning of the substrate in this way would require a slight rearrangement of the residues within the extended loop (190-228) in order to accommodate the steroid D ring and the extended $\mathrm{C} 17$ tail. A previous survey of flavoprotein structures with their bound substrates revealed that the $\mathrm{C}$ atom involved in oxidative attack was positioned $\sim 3.5 \AA$ from the flavin N5 with an angle $(\angle \mathrm{N} 10-$ N5-C) between 96 and $117^{\circ}$ (Fraaije \& Mattevi, 2000). To achieve an angle closer to that reported by Fraaije and Mattevi would result in significant clashes between the substrate and protein atoms involved in catalysis or would require movement of the substrate $\mathrm{O}$ atom away from the position of the conserved water molecule. Our structure reveals a preformed active site utilizing Gly120 to position the flavin N5 lone pair of electrons such that they align appropriately to facilitate efficient hydride transfer from the substrate in the Michaelis complex to generate the reduced flavin during the oxidative half reaction.

The ability to trap crystals in different redox states while maintaining atomic resolution diffraction has provided an unprecedented opportunity to characterize the enzyme active site of cholesterol oxidase at an exquisite level of detail. These studies have given a unique view of a flavin in the reduced state with a hydride transferred to the cofactor and further allow us to delineate important interactions between the protein and the cofactor that facilitate redox chemistry. In particular, a hydrogen-bonding interaction modulates the geometry of the flavin N5 centre and may play an important role in priming the flavin for redox activity.

We thank Anandhi Anandan for assistance with protein expression and purification and Professor Reto Dorta for access to an inert glove box. We also acknowledge the staff at beamlines MX1 and MX2 of the Australian Synchrotron for support with X-ray data collection.

\section{References}

Adams, P. D. et al. (2010). Acta Cryst. D66, 213-221.

Becke, A. D. (1993). J. Chem. Phys. 98, 5648-5652.

Becke, A. D. \& Johnson, E. R. (2005). J. Chem. Phys. 123, 154101.

Berman, H. M., Westbrook, J., Feng, Z., Gilliland, G., Bhat, T. N., Weissig, H., Shindyalov, I. N. \& Bourne, P. E. (2000). Nucleic Acids Res. 28, 235-242.

Cavelier, G. \& Amzel, L. M. (2001). Proteins, 43, 420-432.

Chakrabarti, P. \& Bhattacharyya, R. (2007). Prog. Biophys. Mol. Biol. 95, 83-137.

Chen, L., Lyubimov, A. Y., Brammer, L., Vrielink, A. \& Sampson, N. S. (2008). Biochemistry, 47, 5368-5377.
Compton, S. J. \& Jones, C. G. (1985). Anal. Biochem. 151, 369-374.

Dobbek, H., Gremer, L., Kiefersauer, R., Huber, R. \& Meyer, O. (2002). Proc. Natl Acad. Sci. USA, 99, 15971-15976.

Elias, M., Liebschner, D., Koepke, J., Lecomte, C., Guillot, B., Jelsch, C. \& Chabriere, E. (2013). BMC Res. Notes, 6, 308.

Emsley, P., Lohkamp, B., Scott, W. G. \& Cowtan, K. (2010). Acta Cryst. D66, 486-501.

Faust, A., Niefind, K., Hummel, W. \& Schomburg, D. (2007). J. Mol. Biol. 367, 234-248.

Forneris, F., Heuts, D. P., Delvecchio, M., Rovida, S., Fraaije, M. W. \& Mattevi, A. (2008). Biochemistry, 47, 978-985.

Fraaije, M. W. \& Mattevi, A. (2000). Trends Biochem. Sci. 25, 126-132.

Frisch, M. J. et al. (2009). Gaussian 09. Gaussian Inc., Wallingford, Connecticut, USA.

Fritz, G., Roth, A., Schiffer, A., Buchert, T., Bourenkov, G., Bartunik, H. D., Huber, H., Stetter, K. O., Kroneck, P. M. \& Ermler, U. (2002). Proc. Natl Acad. Sci. USA, 99, 1836-1841.

Ghisla, S. \& Massey, V. (1986). Biochem. J. 239, 1-12.

Goerigk, L. \& Grimme, S. (2014). Wiley Interdiscip. Rev. Comput. Mol. Sci. 4, 576-600.

Grimme, S. (2011). Wiley Interdiscip. Rev. Comput. Mol. Sci. 1, 211-228.

Grimme, S., Ehrlich, S. \& Goerigk, L. (2011). J. Comput. Chem. 32, $1456-1465$

Gustafsson, T. N., Sandalova, T., Lu, J., Holmgren, A. \& Schneider, G. (2007). Acta Cryst. D63, 833-843.

Hall, L. H., Bowers, M. L. \& Durfor, C. N. (1987). Biochemistry, 26, 7401-7409.

Hemel, D. van den, Brigé, A., Savvides, S. N. \& Van Beeumen, J. (2006). J. Biol. Chem. 281, 28152-28161.

Hunter, C. A. (1993). Philos. Trans. A Math. Phys. Eng. Sci. 345, $77-85$.

Hunter, C. A. \& Sanders, J. K. M. (1990). J. Am. Chem. Soc. 112, $5525-5534$

Johansson, R., Torrents, E., Lundin, D., Sprenger, J., Sahlin, M., Sjöberg, B. M. \& Logan, D. T. (2010). FEBS J. 277, 4265-4277.

Johnson, E. R. \& Becke, A. D. (2005). J. Chem. Phys. 123, 024101.

Johnson, E. R. \& Becke, A. D. (2006). J. Chem. Phys. 124, 174104.

Kabsch, W. (2010). Acta Cryst. D66, 125-132.

Karton, A., Tarnopolsky, A., Lamère, J.-F., Schatz, G. C. \& Martin, J. M. L. (2008). J. Phys. Chem. A, 112, 12868-12886.

Kass, I. J. \& Sampson, N. S. (1995). Biochem. Biophys. Res. Commun. 206, 688-693.

Kass, I. J. \& Sampson, N. S. (1998). Biochemistry, 37, 17990-18000.

Kass, I. J. \& Sampson, N. S. (1998). Bioorg. Med. Chem. Lett. 8, $2663-$ 2668.

Khan, H., Barna, T., Bruce, N. C., Munro, A. W., Leys, D. \& Scrutton, N. S. (2005). FEBS J. 272, 4660-4671.

Lario, P. I., Sampson, N. \& Vrielink, A. (2003). J. Mol. Biol. 326, 1635 1650.

Lee, C., Yang, W. \& Parr, R. G. (1988). Phys. Rev. B, 37, 785-789.

Lennon, B. W., Williams, C. H. Jr \& Ludwig, M. L. (1999). Protein Sci. 8, 2366-2379.

Li, X.-L. \& Fu, Y. (2008). J. Mol. Struct. 856, 112-118.

Li, J., Vrielink, A., Brick, P. \& Blow, D. M. (1993). Biochemistry, 32, 11507-11515.

Lyubimov, A. Y., Chen, L., Sampson, N. S. \& Vrielink, A. (2009). Acta Cryst. D65, 1222-1231.

Lyubimov, A. Y., Heard, K., Tang, H., Sampson, N. S. \& Vrielink, A. (2007). Protein Sci. 16, 2647-2656.

Lyubimov, A. Y., Lario, P. I., Moustafa, I. \& Vrielink, A. (2006). Nature Chem. Biol. 2, 259-264.

Mattevi, A., Vanoni, M. A., Todone, F., Rizzi, M., Teplyakov, A., Coda, A., Bolognesi, M. \& Curti, B. (1996). Proc. Natl Acad. Sci. USA, 93, 7496-7501.

McGaughey, G. B., Gagné, M. \& Rappé, A. K. (1998). J. Biol. Chem. 273, 15458-15463. 
Mowat, C. G., Beaudoin, I., Durley, R. C., Barton, J. D., Pike, A. D., Chen, Z.-W., Reid, G. A., Chapman, S. K., Mathews, F. S. \& Lederer, F. (2000). Biochemistry, 39, 3266-3275.

Mowat, C. G., Wehenkel, A., Green, A. J., Walkinshaw, M. D., Reid, G. A. \& Chapman, S. K. (2004). Biochemistry, 43, 9519-9526.

Neese, F. (2012). Wiley Interdiscip. Rev. Comput. Mol. Sci. 2, 73-78.

Nomura, N., Choi, K.-P., Yamashita, M., Yamamoto, H. \& Murooka, Y. (1995). J. Biosci. Bioeng. 79, 410-416.

Pollegioni, L., Wels, G., Pilone, M. S. \& Ghisla, S. (1999). Eur. J. Biochem. 264, 140-151.

Porter, D. J. T. \& Voet, D. (1978). Acta Cryst. B34, 598-610.

Røhr, A. K., Åsmund, K., Hersleth, H. P. \& Andersson, K. K. (2010). Angew. Chem. Int. Ed. 49, 2324-2327.

Rotello, V. M. (1999). Curr. Opin. Chem. Biol. 3, 747-751.

Sampson, N. S. \& Kass, I. J. (1997). J. Am. Chem. Soc. 119, 855862.

Schiffer, A., Fritz, G., Kroneck, P. M. \& Ermler, U. (2006). Biochemistry, 45, 2960-2967.

Sedláček, V., Klumpler, T., Marek, J. \& Kučera, I. (2014). PLoS One, 9, e96262.

Senda, M., Kishigami, S., Kimura, S., Fukuda, M., Ishida, T. \& Senda, T. (2007). J. Mol. Biol. 373, 382-400.

Singh, H., Arentson, B. W., Becker, D. F. \& Tanner, J. J. (2014). Proc. Natl Acad. Sci. USA, 111, 3389-3394.

Stephens, P. J., Devlin, F. J., Chabalowski, C. F. \& Frisch, M. J. (1994). J. Phys. Chem. 98, 11623-11627.
Straaten, K. E. van, Routier, F. H. \& Sanders, D. A. (2012). J. Biol. Chem. 287, 10780-10790.

Sukumar, N., Dewanti, A. R., Mitra, B. \& Mathews, F. S. (2004). J. Biol. Chem. 279, 3749-3757.

Tan, T.-C., Pitsawong, W., Wongnate, T., Spadiut, O., Haltrich, D., Chaiyen, P. \& Divne, C. (2010). J. Mol. Biol. 402, 578-594.

Tarnopolsky, A., Karton, A., Sertchook, R., Vuzman, D. \& Martin, J. M. (2008). J. Phys. Chem. A, 112, 3-8.

Tegoni, M. \& Cambillau, C. (1994). Protein Sci. 3, 303-313.

Waksman, G., Krishna, T. S., Williams, C. H. Jr \& Kuriyan, J. (1994). J. Mol. Biol. 236, 800-816.

Walsh, J. D. \& Miller, A.-F. (2003). J. Mol. Struct. 623, 185-195.

Weigend, F. \& Ahlrichs, R. (2005). Phys. Chem. Chem. Phys. 7, $3297-$ 3305.

Winn, M. D. et al. (2011). Acta Cryst. D67, 235-242.

Wohlfahrt, G., Witt, S., Hendle, J., Schomburg, D., Kalisz, H. M. \& Hecht, H.-J. (1999). Acta Cryst. D55, 969-977.

Yamashita, M., Toyama, M., Ono, H., Fujii, I., Hirayama, N. \& Murooka, Y. (1998). Protein Eng. Des. Sel. 11, 1075-1081.

Yin, Y., Liu, P., Anderson, R. G. \& Sampson, N. S. (2002). Arch. Biochem. Biophys. 402, 235-242.

Yin, Y., Sampson, N. S., Vrielink, A. \& Lario, P. I. (2001). Biochemistry, 40, 13779-13787.

Yue, Q. K., Kass, I. J., Sampson, N. S. \& Vrielink, A. (1999). Biochemistry, 38, 4277-4286.

Zheng, Y.-J. \& Ornstein, R. L. (1996). J. Am. Chem. Soc. 118, 94029408. 